\title{
Verificación de las características metrológicas de un contador de flujo Coriolis utilizado en transferencia fiscal
}

\section{Verification of metrological characteristics of a Coriolis flow meter used in fiscal transfer}

\author{
Barrera-García Aníbal \\ Universidad de Cienfuegos, Cuba \\ Departamento de Ingeniería Industrial \\ Correo: abarrera@ucf.edu.cu \\ Hernández-Santana Midiala \\ CUVENPETROL S.A. \\ Unidad de Negocios Refinería Cienfuegos, Cuba \\ Correo: mhernandez@cuvenpetrol.cu \\ Botana-Beltrán Leandro Oscar \\ Universidad de Cienfuegos, Cuba \\ Departamento de Física \\ Correo: obotana@ucf.edu.cu
}

\author{
Martínez-Hernández Tanayi \\ Departamento de Ingeniería Industrial \\ Universidad de Cienfuegos, Cuba \\ Carrasco-Padrón Oleiny Omar \\ Departamento de Ingeniería Industrial \\ Universidad de Cienfuegos, Cuba \\ Correo: ocarrasco@ucf.edu.cu
}

\section{Resumen}

En el presente artículo se describe un procedimiento para la evaluación de la calidad de las mediciones. La propuesta garantiza la evaluación y mejora de la gestión de las mediciones de flujo, utilizando técnicas relacionadas con la metodología seis sigma. Esto se aplica en una empresa refinadora de petróleo, específicamente en el proceso de recepción, almacenamiento, manipulación y entrega de gas licuado del petróleo. Para la recopilación y procesamiento de la información se utilizaron técnicas tales como: trabajo con expertos, trabajo en equipo, estudio de repetibilidad, estabilidad, linealidad, diseño experimental. Se hace uso de herramientas clásicas de la calidad y de gestión de procesos. Como resultados fundamentales de la investigación se identificaron las principales debilidades desde el punto de vista metrológico, se evaluó el estado de las características metrológicas del flujómetro másico Coriolis utilizado en la entrega de gas licuado del petróleo regular por cargadero de camiones. Se efectúa un estudio de repetibilidad y reproducibilidad basado en el diseño experimental en dicha operación. Por último, se proponen un conjunto de acciones que contribuyen a la mejora de la calidad de las mediciones, así como en su gestión.

Descriptores: repetibilidad, reproducibilidad, mediciones, instrumentos, estabilidad.

\begin{abstract}
This article describes a procedure for the evaluation of the quality of measurements. The proposal guarantees the evaluation and improvement of the management of flow measurements, using techniques related to the six sigma methodology. This is applied to an oil refining company, specifically in the process of receiving, storing, handling and delivering liquefied petroleum gas. For the collection and processing of information, techniques such as: working with experts, teamwork, repeatability study, stability, linearity, experimental design were used. It makes use of classic quality tools and process management. As fundamental results of the investigation, the main weaknesses were identified from the metrological point of view, the state of the metrological characteristics of the Coriolis mass flowmeter used in the delivery of regular liquefied petroleum gas by truck loading was evaluated. A repeatability and reproducibility study is carried out based on the experimental design in said operation. Finally, a set of actions are proposed that contribute to the improvement of the quality of the measurements, as well as their management.
\end{abstract}

Keywords: repeatability, reproducibility, measurements, instruments, stability. 


\section{INTRODUCCIÓN}

El nuevo enfoque integral de la calidad brinda un sistema de gestión que asegura que las organizaciones satisfagan los requerimientos de los clientes, y a su vez, hagan uso racional de los recursos, asegurando su máxima productividad. Así mismo permite desarrollar en la organización una fuerte ventaja competitiva como es la cultura del "mejoramiento continuo" con un impacto positivo en la satisfacción del cliente, del personal y un incremento de la productividad. Autores como Xiaofen (2013), Myszewski (2013) y Antony (2013), aseguran que los métodos de calidad ahora son el pilar sobre el que se apoya toda empresa para garantizar su futuro. Quién no se encuentre en proceso de normalizar su empresa, implantar un sistema de calidad y obtener la certificación, no tiene futuro. Según el criterio de los autores Galvania y Carpinettib (2013) e Yvonne (2013), con la ayuda de iniciativas como seis sigma, hay mayor aceptación de la importancia de los análisis de datos, definiendo y justificando el pensamiento estadístico y su importancia para el movimiento de calidad.

Las organizaciones que desarrollan sistemas de gestión de la calidad frecuentemente se enfrentan a la necesidad de profundizar sus conocimientos relacionados con la metrología. La investigación metrológica no es solo un factor esencial para garantizar la calidad de la producción, además contribuye a un mayor desarrollo en áreas como la salud, la seguridad y la energía. La European Association of National Metrology Institutes (2013) plantea que la metrología es verdaderamente esencial para asegurar la calidad en la normalización.

En la industria refinadora de petróleo donde se desarrolla la investigación se encuentra implementada la NC ISO 10012:2007. En esta entidad se trabaja en la aplicación de la filosofía de mejora seis sigma en parte de sus procesos, pero aún no se ha implementado en la gestión de las mediciones. En la misma se produce cierta variedad de productos, en cuyos procesos intervienen un grupo de instrumentos y procedimientos de trabajo que repercuten en la calidad final del producto. Uno de ellos es el Gas Licuado del Petróleo (GLP), que por sus características físicas y químicas es un combustible con cierta dificultad para su control. La industria petrolera emplea innumerables equipos de medición, pero no todos cuentan con el aseguramiento metrológico necesario para la transferencia fiscal, conllevando a pérdidas económicas.

El control metrológico en este tipo de mediciones tiene como principal propósito que la exactitud durante las transacciones se encuentre entre los límites internacionalmente establecidos, representando seguridad y confianza en los resultados del proceso de medición (Hernández, 2012a), así como impulsar la toma de decisiones con base en fundamentos correctos, y llevar un control apropiado del inventario a la hora que se presente la fiscalización de las autoridades competentes. La transferencia de custodia se debe realizar en el marco de la metrología legal, constituyendo las mediciones de volumen y flujo como la base del control de las operaciones de transferencia de custodia y de mediciones fiscales de la industria objeto de estudio.

En este tipo de industria se emplean los medidores de flujo Coriolis y se emplean transacciones comerciales, donde la medición del producto vendido se puede dispensar en unidades de masa, debido a que la masa de un fluido no se ve influenciada por variables físicas como presión, temperatura o densidad. Los autores Germanier y López Carrizo (2006), Wang y Baker (2014) y Franco et al. (2014) definen las principales ventajas de los medidores de flujo Coriolis en las transferencias de custodia:

- Menor mantenimiento y costo de inventario

- Mayor producción y caudal a medir

- Mayor mantenimiento de la exactitud

- Reducción de costos de instalación

- Bidireccionalidad

- Información en tiempo real del medidor para el sistema supervisor o de adquisición de datos

- Amplia experiencia y utilización en aplicaciones de la industria del petróleo

- Alta exactitud: error máximo permisible (0.1-0.3\%) en correspondencia con las R OIML 117

- Cuentan con computadoras de flujo que corrigen a temperatura de referencia

- Medición de temperatura y densidad en tiempo real

Un ejemplo de mediciones fiscales son las lecturas de volúmenes y masas de los medidores de tanques, que son la base para el cálculo y la facturación, por lo que deben ser exactas para que la desviación no provoque consecuencias negativas relevantes. Esto tiene especial aplicación a efectos contables, como en el control/verificación de transferencia, la gestión de inventarios y el control de pérdidas (Chang, 2007).

En la empresa refinadora donde se realizó el estudio, la trazabilidad de los flujómetros másicos (Coriolis) utilizados para el servicio de transferencia fiscal se realizó por comparación con un Patín Máster Meter, siendo limitada su trazabilidad en el país. Por tanto, es necesario realizar estudios de repetibilidad y estabilidad del instrumento para demostrar que sus valores son constantes en el tiempo, que es capaz de repetir y que sus mediciones son confiables. 
El objetivo de este trabajo es desarrollar y probar un procedimiento para la evaluación de calidad de las mediciones de flujo en procesos de transferencia fiscal en la entrega de GLP. Con el mismo se obtuvo como resultado la evaluación de las características metrológicas de un flujómetro Coriolis utilizado en transferencia fiscal, así como propuestas de mejoras para el sistema de medición analizado.

\section{Desarrollo}

El procedimiento empleado se muestra en la figura 1, se organiza metodológicamente en cinco etapas y diecisiete pasos. Para su elaboración se utilizaron criterios de los autores Gutiérrez y de la Vara (2009), Correa y Burgos (2007), Gibbons et al. (2012), Guadalupe (2008) e ISO 13053:2011, entre otros.

\section{DisCuSIÓN Y ANÁLISIS DE LOS RESULTADOS}

Para la aplicación se tomó como objeto de estudio una empresa refinadora de petróleo, perteneciente al sector de la industria petroquímica.

\section{EtAPa I: DefiniR}

\section{Paso 1: Descripción General Del PROBlema}

La empresa refinadora de petróleo que se seleccionó para la investigación, cuenta con un Sistema de Gestión de Calidad basado en la NC ISO 9001:2008 (transitando al estándar NC ISO 9001:2015) y posee certificación Lloyd Register. Este sistema se encuentra interrelacionado con la norma NC ISO 10012: 2007, específicamente en su apartado 7.6, donde se exponen los requisitos relacionados con el análisis y mejora del sistema de gestión de las mediciones, este requisito se aplica a cualquier sistema de gestión. Por tanto, los procesos de medición deben considerarse como procesos específicos, cuyo objetivo es apoyar la calidad de los productos elaborados por la organización, aspecto que no se evalúa en su totalidad en la organización. Estos elementos constituyen la principal razón que conlleva a la necesidad de evaluar la calidad de las mediciones en los procesos que se desarrollan en dicha industria.

\section{Paso 2: MAPEO Del PROCESO}

El proceso de instrumentación y metrología se encuentra subordinado a la Gerencia de Calidad y la Gerencia de Automática, Informática y Telecomunicaciones (AIT). Se cuenta con el diagrama de proceso y ficha correspondiente.

\section{Paso 3: Diagnóstico del proceso}

Se define para cada uno de los instrumentos que integran el proceso de medición, el período de calibración y verificación, establecido por el Decreto Ley Núm. 183 de febrero de 1998 y regulado por la Disposición General DG-01 “Instrumentos de medición sujetos a la verificación obligatoria y a aprobación de modelo según los

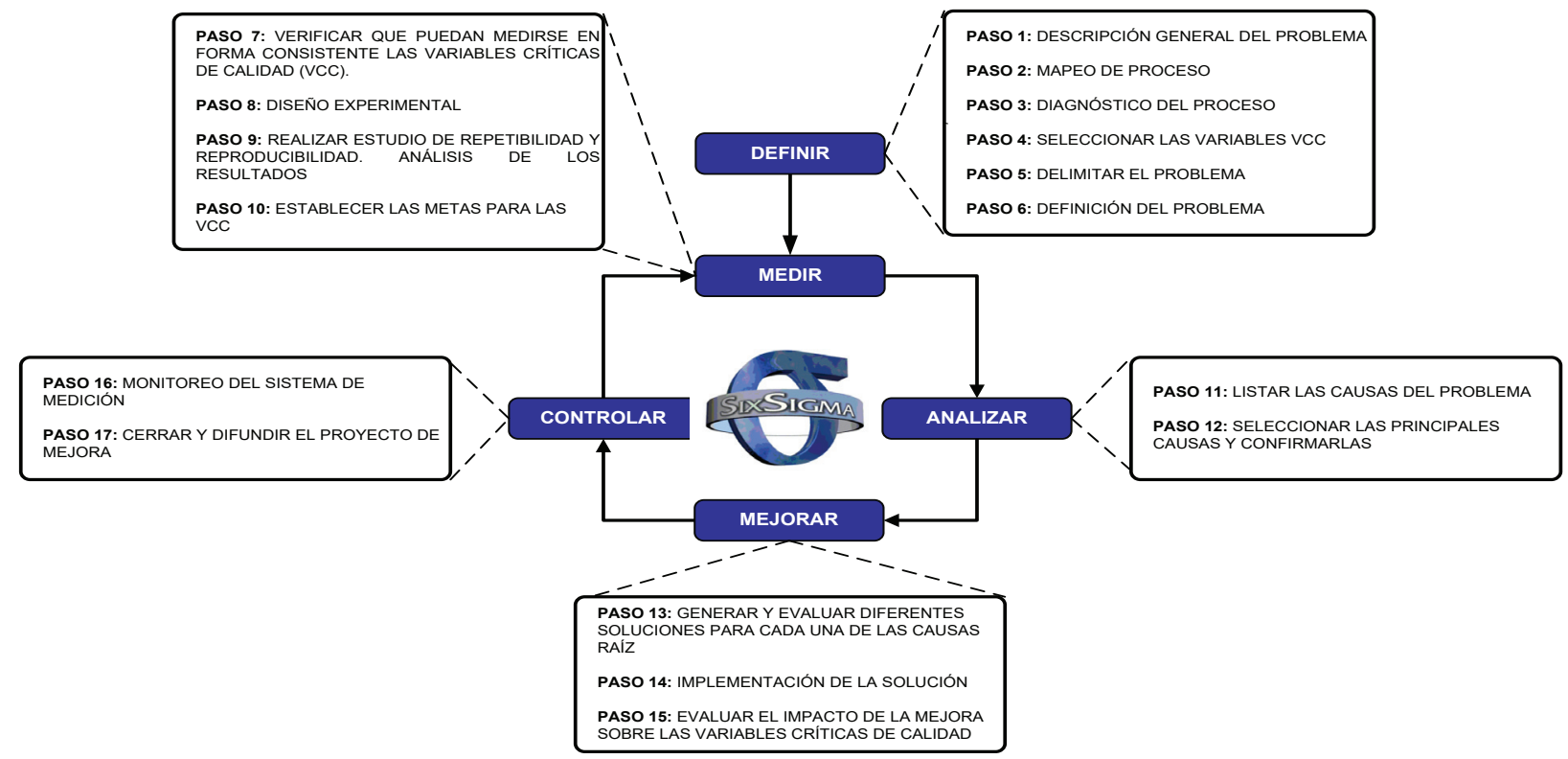

Figura 1. Procedimiento para la mejora de la gestión de las mediciones a partir de la utilización de la metodología seis sigma 
campos de aplicación donde serán utilizados". Las principales dificultades en la entidad se centraron en:

- La extensión del período de extracción para la calibración o verificación del instrumento

- Falta de trazabilidad en algunos instrumentos

- Ausencia de estudios para conocer la calidad en el sistema de gestión de las mediciones

\section{Paso 4: Seleccionar las Variables críticas para la CAli- DAD (VCC)}

Para definir las variables críticas de calidad de mayor impacto a los clientes del proceso de instrumentación y metrología, se realizó una sesión con el especialista principal en metrología y personal de amplia experiencia en la temática, de lo que resultaron las siguientes variables:

- Período de verificación y calibración

- Tiempo de ciclo de salida y reposición del instrumento al proceso

- Calidad en los sistemas de mediciones

Para lograr la prioridad de estas variables se efectuó una valoración de cada una de ellas, donde se definió como variable crítica la calidad en los sistemas de medición.

\section{Paso 5: Delimitar el problema}

El GLP es el producto que presenta mayor complejidad en la medición y control de inventarios por almacenarse en recipientes presurizados, así como diversidad en su comercialización. Su entrega se realiza a través de básculas Crisplant y flujómetros Coriolis Endress+Hauser con error máximo permisible de $0.25 \%$. A estos últimos se les debe avalar la calidad en sus mediciones mediante estudios estadísticos. Las básculas Crisplant que se utilizan en el llenado de cilindros tienen trazabilidad en el país, pues se pueden verificar y calibrar sin dificultad, mientras que a los flujómetros Coriolis se le garantiza su trazabilidad con un Patín Máster Meter, para el que no hay aseguramiento metrológico. Por lo anterior se seleccionó el proceso de recepción, almacenamiento, manipulación y entrega de GLP.

En la empresa se adquirió una estación móvil (ver figura 2a) para garantizar la trazabilidad de los flujómetros Coriolis, pero los patrones que están montados tienen validez solo por dos años, estando próximo a su vencimiento. Por esta razón, se decidió efectuar un estudio de repetibilidad y estabilidad del instrumento para indicar que sus valores son constantes en el tiempo y que es capaz de repetir; demostrando que sus mediciones son confiables. Por tanto, es a través de la calidad de la medición de los flujómetros Coriolis (figura $2 b$ ) y la carga de GLP por roto gauge, que se analizará la calidad del resultado del sistema de medición.

\section{PASO 6: DeFINICIÓN DEL PROBLEMA}

El proceso de entrega de GLP regular por cargadero de camiones rígidos y articulados, es el que se realiza con mayor frecuencia, donde existe la mayor parte de las quejas, reclamaciones e inconformidades; dadas por las incongruencias entre la cantidad entregada y recibida. La entrega se hace volumétricamente, según la capacidad del carro por un roto gauge que no tiene trazabilidad. La política de comercialización definida en el país es la venta por volumen, este puede variar notablemente por influencia de las condiciones físicas de presión, temperatura y densidad, donde lo más conveniente es la venta en masa, que no se ve afectada por estas variables. En el cargadero de camiones se encuentran instalados flujómetros Coriolis, que son líderes en la medición de caudales másicos, donde a través de la calidad de la medición de estos instrumentos se estudiará la calidad del resultado del sistema de medición.

\section{EtAPA II: Medir}

PASO 7: VERIFICAR QUE PUEDAN MEDIRSE EN FORMA CONSISTENTE LAS VARIABLES CRÍTICAS DE CALIDAD

Todos los instrumentos que intervienen en el proceso tienen aseguramiento metrológico, excepto el roto gauge, que se utiliza para la medición del porciento de la capacidad por nivel. Este recibe servicio de mantenimiento y comprobación de su alineación.

Se definieron los elementos que intervienen en la variabilidad del equipo que se utiliza en esta actividad (Gutiérrez y de la Vara, 2009). A continuación se muestra el resultado para el flujómetro Coriolis A4070802000 Endress+Hauser modelo 84F80-AABSAG4AAAAM.

\section{- $\quad$ Calibración y verificación}

Por intervenir en transferencia fiscal y utilizarse para la comercialización de combustible, requiere verificarse según la Disposición General DG-01. La verificación del instrumento mencionado se realizó por el Centro Territorial de Metrología, amparada por el Certificado Núm. 661502, con fecha de 12 de marzo de 2013. Este es trazable al Máster Meter No. D8092402000 y conforme a la OIML R 117:2007. 

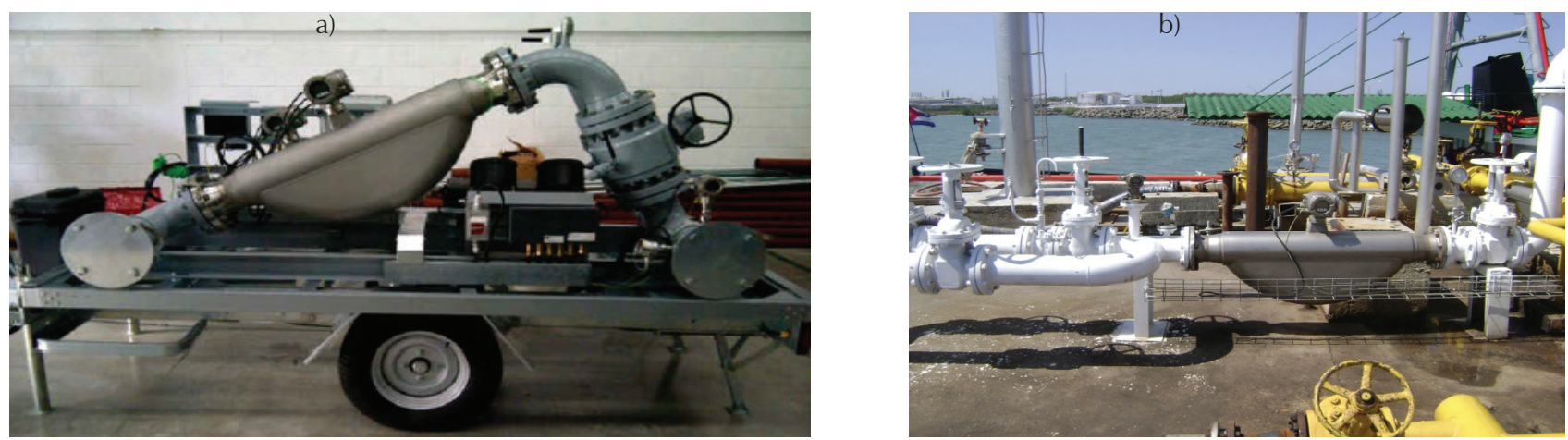

Figura 2. a) Estación móvil (Patín máster meter), b) Flujómetro Coriolis utilizado en la transferencia

\section{- Estabilidad}

Para conocer la estabilidad del instrumento se tomaron datos desde marzo hasta diciembre de 2013. En este caso, Gutiérrez y de la Vara (2009) proponen utilizar la carta de individuales y la carta de medias móviles exponencialmente ponderadas (EWMA), debido a que se generan pocos resultados por día, graficando la suma ponderada de las medias de los subgrupos observados hasta el tiempo de inspección, que asigna pesos decrecientes a las medias anteriores. Con el procesamiento de los datos en el software Statgraphics Centurión se obtuvo la carta EWMA, que se muestra en la figura 3.

Se aprecia que no hay tendencias, ni ningún otro patrón especial, ni puntos fuera de los límites de control. Al calcular la estabilidad se puede decir que es buena (menor a 2\%). Por tanto, el proceso ha estado funcionando de manera estable y se encuentra bajo control estadístico.

\section{- $\quad$ Repetibilidad}

La repetibilidad se analizó como la variación asociada con las mediciones repetidas de dicho instrumento respecto al patrón de referencia Máster Meter. Este contador de flujo posee un error máximo permisible de $\pm 1 \%$, dado en el Certificado de Verificación Núm. 661502.

Se inició el estudio tomando seis mediciones del flujómetro respecto a su patrón. Luego se tomaron los datos y surgió la necesidad de conocer si la cantidad de observaciones eran suficientes, esto se realizó a partir de las curvas características (Montgomery, 1991), concluyendo que deben ser al menos ocho observaciones.

Para ello, se conectó en serie el Máster Meter con el Meter Prueba instalado en el cargadero de pailas. Para el cálculo del error se utilizó la expresión dada en la NC 918: 2012 "Metros Contadores para líquidos diferentes del agua. Métodos y equipos para la verificación":

$E_{c}=$ Volumen medido por el contador que se comprueba Volumen medido por el instrumento patrón

donde

$E_{c}=$ Error de indicación

El error de indicación relativo $\left(E_{r}\right)$ expresado en \% se calcula con la siguiente ecuación

$E_{r}=\frac{E_{C}}{V_{p}} \times 100$

donde

$V_{p}=$ Volumen medido por el instrumento patrón

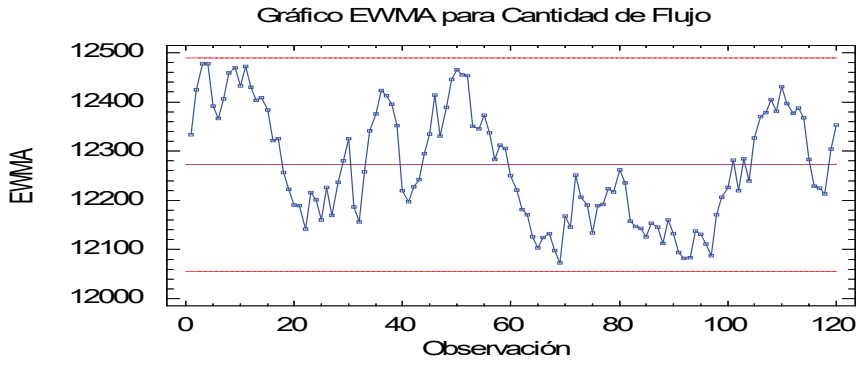

Figura 3. Carta EWMA para el volumen de GLP medido por el flujómetro Coriolis desde marzo hasta diciembre de 2013 
Mientras el error de repetibilidad $\left(\mathrm{E}_{\text {rep }}\right)$ expresado en \% se calculó por la siguiente fórmula

$$
E_{\text {rep }}=\frac{\text { valor máximo }- \text { valor mínimo }}{\text { valor mínimo }} \times 100
$$

El error de repetibilidad debe ser menor o igual que 1/3 del error máximo permitido para el contador. Los resultados se muestran en la tabla 1.

Como el error máximo permisible es de $1 \%$ según el certificado de verificación 661502, se obtuvo

$\% E_{\text {rep }}=0.33$

Luego se calculó el error de repetibilidad del equipo $\% E_{\text {rep }}=\% E_{\text {rep mayor }}-\% E_{\text {rep menor }}=0.18 \%$

Finalmente se comparó este valor con el \% de repetibilidad máximo del equipo, resultando que el instrumento posee buena repetibilidad.

\section{- $\quad$ Linealidad}

Para determinar la exactitud a lo largo del rango de operación del flujómetro Coriolis (linealidad), se tomaron los datos de la verificación realizada el 12 de marzo del 2013. Se eligieron tres flujos en el rango de operación, Flujo Mínimo, Flujo Medio y Flujo Máximo. Estos se midieron tres veces en un mismo intervalo de tiempo bajo las mismas condiciones. Con los resultados obtenidos se realizó el análisis de linealidad del equipo, que se muestra en la siguiente gráfica.

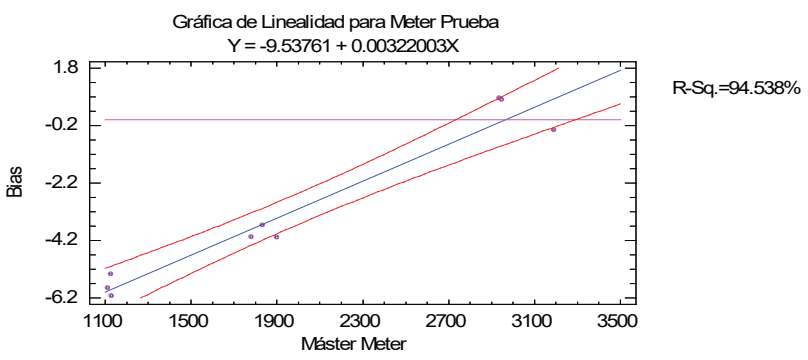

Figura 4. Gráfica de linealidad para Meter Prueba

$\mathrm{Al}$ analizar la figura anterior se tiene que basado en un total de nueve mediciones, el sesgo medio estimado del dispositivo es -3.11556 unidades. Esto representa $0.150 \%$ de la variación del proceso, donde este es menor que $1 \%$, especificado como 2079.57 unidades. La linealidad, o cambio de sesgo a lo largo del rango de variación del instrumento, representa $0.322 \%$ de ese rango. Se observa que todos los puntos se encuentran en el límite de confianza para la línea de regresión.

Existe buena relación lineal entre las mediciones del Meter Prueba y el Máster Meter, donde el $\mathrm{R}^{2}$ se ajusta $94.538 \%$, por lo que se puede decir que el instrumento tiene buena linealidad.

Se construyó el gráfico de exactitud (figura 5), mostrando la estimación de la exactitud del proceso de medición.

Las bandas sobre el gráfico muestran la estimación de los límites de predicción para observaciones individuales, en el mismo se detectó que no hay valores atípicos, debido a que no existen puntos fuera de los límites de predicción, por tanto posee buena exactitud el instrumento.

Tabla 1. Estudio de repetibilidad del flujómetro respecto al patrón Máster Meter

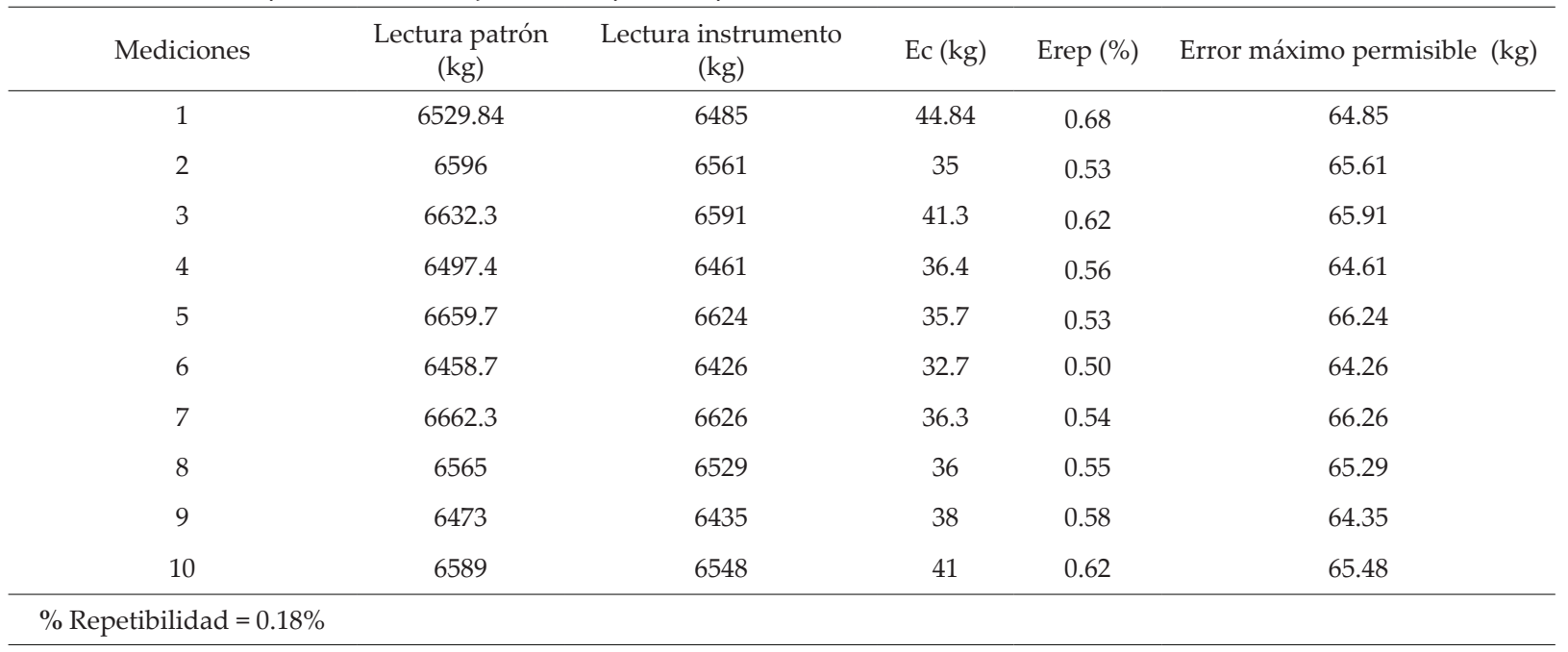


Del estudio anterior se concluyó que las mediciones efectuadas mediante el flujómetro Coriolis tienen calidad con una aceptable variabilidad.

En la entrega de GLP por camiones rígidos interviene además el operario, que es uno de los elementos que aporta mayor variabilidad en el resultado de la medición de la cantidad entregada, debido a que cierra la válvula a 90\% de llenado por roto gauge del camión, cuando comienza a salir GLP en fase líquida. Para analizar esta problemática se decidió realizar un estudio de repetibilidad y reproducibilidad (R\&R) en la entrega de GLP regular por camiones rígidos.

\section{PASO 8: DiseÑo EXPERIMENTAL}

El diseño experimental consiste en planear y realizar un conjunto de pruebas con el objetivo de generar datos que, al analizarse estadísticamente, proporcionan evidencias objetivas que permiten responder las interrogantes planteadas por el experimentador sobre determinada situación. El análisis de varianza (ANOVA) es la técnica central en el análisis de datos experimentales, que consiste en separar la variación total observada en cada una de las fuentes que contribuye a la misma.

Para seleccionar el tipo de diseño experimental a utilizar se definieron las fuentes fundamentales de variación durante la carga de GLP: el método y la habilidad de los operarios, equipo utilizado, medio ambiente y mediciones. De ellas, se eligió la habilidad del operario, ya que es la fuente de mayor peso de acuerdo con estudios previos realizados por los autores Hernández (2012b) y Machado (2013) en la industria refinadora de petróleo analizada. Se quiere entonces determinar el efecto de esta variable sobre los resultados de las mediciones de la cantidad de GLP despachado, cuando se mantienen controladas las demás fuentes, de este modo no solo se conoce la variabilidad en los resultados de las mediciones, sino también la confiabilidad en ellas.

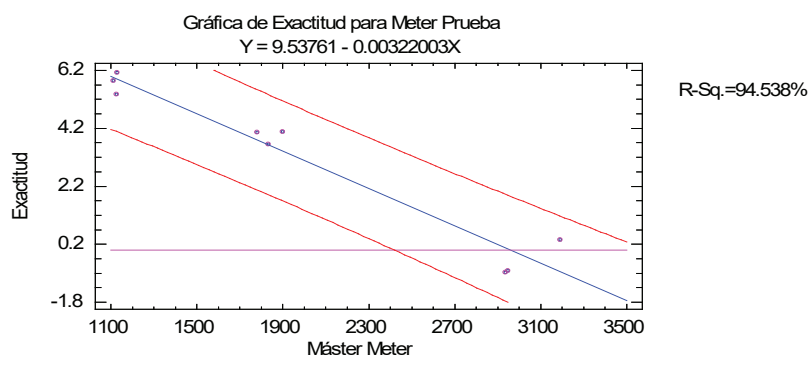

Figura 5. Gráfica de exactitud para Meter Prueba
Las variables de interés en el estudio son la habilidad del operario y cantidad de GLP cargada, donde la primera es una variable independiente, controlable y cualitativa con cuatro niveles: A, B, C y D. El resto de las variables de respuesta son cuantitativas y continuas.

- Determinar el modelo estadístico

Se decidió seleccionar el modelo unifactorial, que se caracteriza por la presencia de una variable controlable en el experimento y la ausencia de manipulación de variables incontrolables en la toma de datos. El modelo unifactorial de efectos aleatorios que se desarrolló consideró el factor controlable la habilidad del operario y como variable de respuesta la cantidad de GLP despachada.

\section{- Determinar tamaño muestral}

Para determinar el tamaño muestral se siguió el mismo procedimiento realizado anteriormente. Se utilizaron las curvas características (Montgomery, 1991) y se identificó $\beta$ con los datos recopilados. Para una potencia de $95 \%$ se tiene que 6 réplicas son insuficientes. Se decidió por parte del equipo de trabajo realizar un total de cuatro réplicas más por operario, para obtener mejores resultados.

Paso 9: Realizar estudio de RePeTIBILIDAD y ReProducibiLIDAD. ANÁLISIS DE LOS RESULTADOS

En este caso son 80 observaciones (4 operadores $\times 20$ réplicas), denominados con la simbología $\mathrm{A}, \mathrm{B}, \mathrm{C}$ y $\mathrm{D}$, con dos repeticiones cada uno, en dos períodos de tiempo. Cada uno de ellos tiene diez medidas alternadas. VERIFICACIÓN DE LOS SUPUESTOS DEL MODELO

En este paso se describe la forma de verificar los supuestos del modelo estadístico. Estos supuestos son (Gutiérrez y de la Vara, 2008):

- Los errores deben tener una distribución normal

- Los errores deben ser independientes

- Las varianzas de los tratamientos deben ser iguales

El primer supuesto considerado es el de normalidad, que se analizó a partir del sesgo y la curtosis estandarizada, debiendo oscilar entre -2 y 2 para que los residuos sigan distribución normal. Este supuesto se cumplió, debido a que los errores oscilan en el rango especificado.

El segundo supuesto considerado es la independencia de los errores. En la figura 6 se presenta el gráfico de los residuales. 
En el gráfico anterior se observa una nube de puntos que no tienen una tendencia definida, los residuos se encuentran distribuidos en forma aleatoria, esto indica independencia de los residuos y por tanto independencia en las observaciones.

El tercer supuesto es el de igualdad de varianzas, donde se empleó para su verificacón la prueba de Bartlett. Con esta prueba se busca comprobar la siguiente hipótesis:

$\mathrm{H}_{\mathrm{o}}: \sigma_{A}=\sigma_{B}=\sigma_{C}=\sigma_{D}=\sigma$

$\mathrm{H}_{\mathrm{A}}: \sigma_{i} \neq \sigma_{j}$ para algún $i \neq j=A, B, C, D$

El estadístico calculado evalúa la hipótesis de que la desviación estándar de la cantidad de GLP medido dentro de cada uno de los 4 niveles (operadores) es la misma. Puesto que el valor-P es mayor o igual que 0.05 , no existe una diferencia estadísticamente significativa entre las desviaciones estándar, con un nivel de 95.0\% de confianza. Por lo tanto, si $0.05<0.57047$, entonces se acepta la hipótesis nula, es decir, se cumple el supuesto de igualdad de varianza.

\section{ANÁLISIS DE LOS RESULTADOS}

Los resultados del ANOVA y el planteamiento de pruebas de hipótesis actúan como una buena herramienta para la toma de decisiones acerca de la significancia del efecto del factor (destreza de los operarios) sobre la variable respuesta. La prueba de hipótesis planteada dentro del estudio es:

$\mathrm{H}_{\mathrm{o}}=$ No hay diferencias significativas en la variabilidad existente entre los operadores.

$\mathrm{H}_{\mathrm{A}}=$ Hay diferencias significativas en la variabilidad existente entre los operadores.

$\mathrm{Al}$ analizar el resultado del método ANOVA para el modelo unifactorial de efectos aleatorios, se evidencia que no existe diferencia estadísticamente significativa

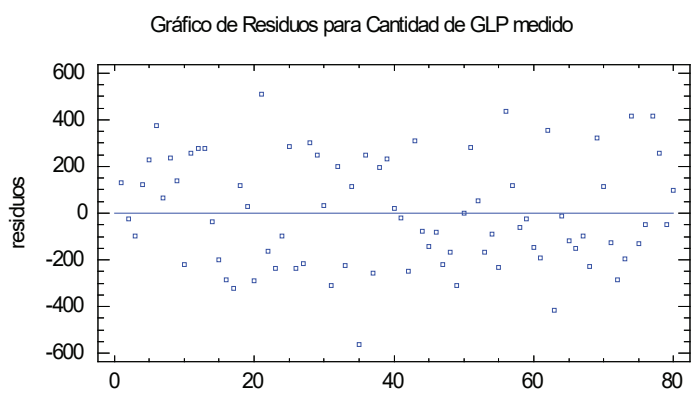

Figura 6. Gráfica de residuales vs observaciones para el estudio en la variabilidad de las mediciones entre los operadores, ni dentro de los tratamientos.

Puesto que el valor-P es mayor que 0.05 , no existe una diferencia significativa en la variabilidad de las mediciones entre los operadores, con un nivel de $95.0 \%$ de confianza. Por lo tanto, si $0.05<0.8342$, entonces se acepta la hipótesis nula.

Los datos obtenidos del estudio se procesaron nuevamente utilizando el software Minitab 15.0, para de esta forma conocer la variabilidad del sistema de medición mediante el ANOVA para el estudio R\&R largo. Este método permite identificar y cuantificar todas las fuentes de variación presentes en el estudio $R \& R$.

La variabilidad es igual a $100 \%$, por tanto, el sistema de medición se considera no aceptable. El número de categorías distintas (nc) que pueden distinguirse confiablemente por el sistema de medición analizado en este estudio es igual a 1. Normalmente, este número debe ser al menos 5 (Gutiérrez y de la Vara, 2009). De la varianza total, $100 \%$ se debe al instrumento (repetibilidad), corroborándose de forma gráfica en la figura 7.

Se concluyó que entre los operadores no hay similitud en sus mediciones, incluso el mismo operador utilizando el mismo carro, declara mediciones diferentes, demostrando que existen diferencias en los resultados debido a la variabilidad en el método de medición e indicando que no es el adecuado. En estas mediciones inciden otros factores, como son: apreciación del operador al determinar la salida de GLP en fase líquida por el roto gauge a $90 \%$, variación de la temperatura y con ello la densidad. Por tanto, se deben proponer acciones que mejoren las desviaciones existentes.

PASO 10: ESTABLECER LAS METAS PARA LAS VARIABLES CRítICAS DE CALIDAD

Teniendo en cuenta la situación para la variable crítica de calidad (calidad de las mediciones en la entrega de GLP regular por camiones cisternas), se planteó como meta realizar la entrega a través del flujómetro Coriolis en masa, para reducir el porcentaje de variación total en la entrega por correcciones (temperatura, densidad y presión) y utilizar la carga a $90 \%$ por roto gauge como técnica de seguridad. 


\section{R\&R del sistema de medición (ANOVA) para Resultados}

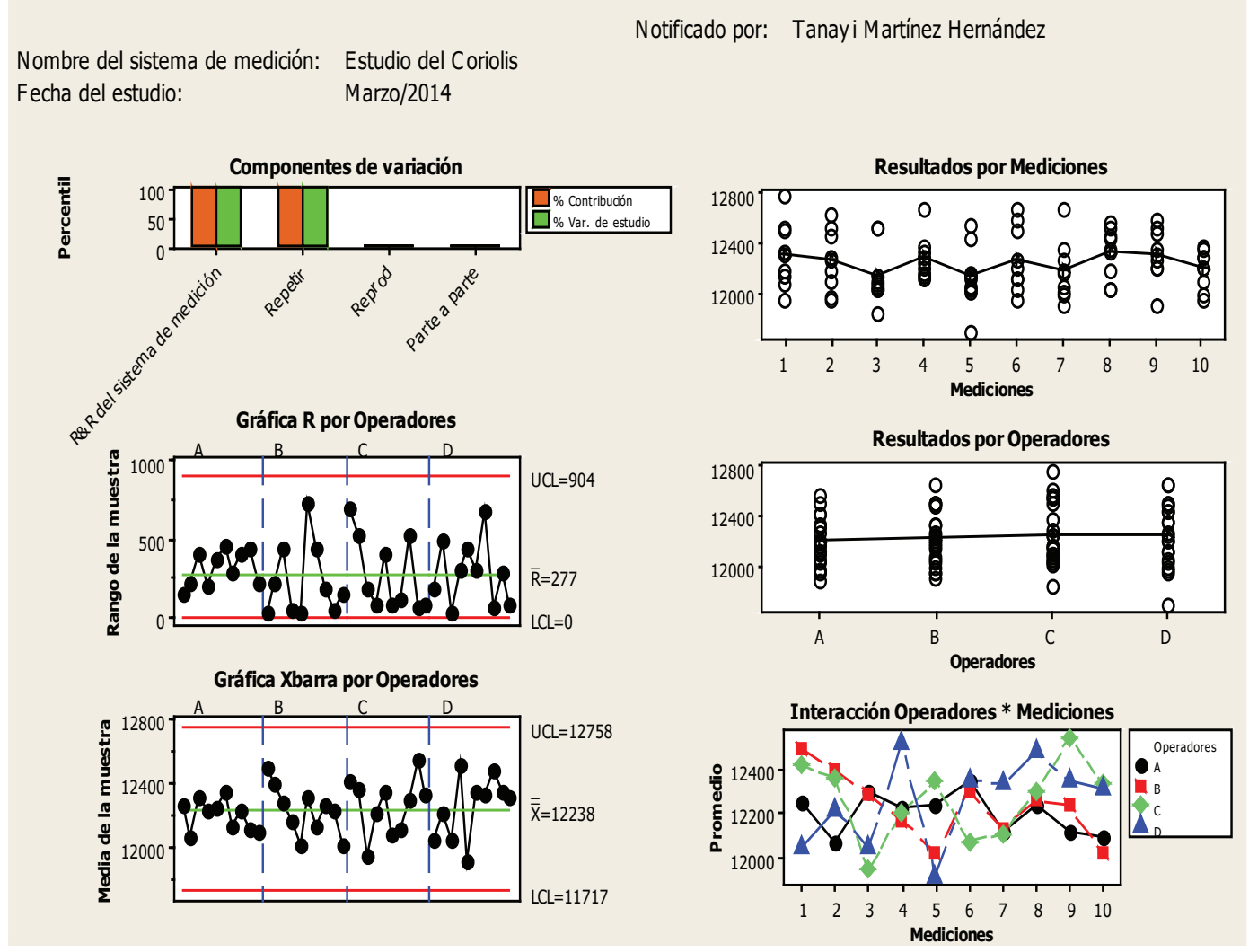

Figura 7. R\&R del sistema de medición (ANOVA) para la entrega de GLP regular por camiones rígidos

\section{Etapa III: Analizar las causas raíz}

PASO 11 y 12: Listar LAS CAUSAS DEL PROBLEMA, SELECCIONAR LAS PRINCIPALES Y CONFIRMARLAS

Después de haber identificado los problemas existentes, se hizo un análisis de las causas. Este análisis se dividió en dos aspectos:

- Preparación del diagrama causa-efecto: Se realizó el análisis de causa y efecto para determinar las posibles causas que influyen en la variación del resultado de las mediciones en la entrega de GLP regular por camiones cisternas. Dicho diagrama se construyó en una sesión de tormenta de ideas de conjunto con el equipo de mejora (Martínez, 2014).

- Planteamiento de las hipótesis y verificación de las causas más probables: El equipo de mejora revisó las causas posibles y seleccionó las más probables.

\section{Etapa IV: MejORAR}

En esta etapa se propusieron, implementaron y evaluaron las soluciones a las causas raíces detectadas, demostrando que las soluciones propuestas resuelven el problema y llevan a las mejoras buscadas.

\section{Paso 13: Generar y eValuar diferentes soluciones para CADA UNA DE LAS CAUSAS RAÍCES}

Se generaron un grupo de soluciones para las causas raíces detectadas $\mathrm{u}$ oportunidades de mejora. Por consenso del equipo de trabajo, se decidió priorizar las siguientes:

- Realizar la carga en masa, a un valor promedio para cada uno de los carros, utilizando como base la estadística del año 2013, debido a que en este período el instrumento mantuvo sus características metrológicas. 
- Garantizar flujo máximo para no afectar las características del flujómetro, mantener el régimen de bombeo y tiempo de carga estable.

- Realizar la verificación del flujómetro según el plan de verificación/calibración.

\section{PAso 14: IMPLEMENTACIÓN DE LA SOLUCIÓN}

Para realizar la implementación de las soluciones se diseñaron los planes de mejora correspondientes. Orientado a la revisión de las medidas propuestas se diseñó un plan de control, según previo análisis en sesión de trabajo entre los integrantes del equipo (Martínez, 2014).

\section{Paso 15: Evaluar el impacto de la mejora sobre las Va-} RIABLES CRÍTICAS DE CALIDAD

Dentro de las medidas que se implementaron se encuentra la verificación del flujómetro. Este se verificó por la Oficina Territorial de Normalización bajo las mismas condiciones en que se realizó durante el año 2013, amparada por el Certificado de Verificación Núm. 661301. Se comprobó que dicho instrumento mantiene sus características metrológicas. Se evidenció que el porciento de error disminuye en la última verificación, demostrándose que el instrumento continuaba manteniendo sus características metrológicas.

Respecto al régimen de bombeo, se estableció como condicionante en la Instrucción RF-DMCP-IT-23-01 la observación de la presión en el manómetro, en la línea de entrega de GLP por cargadero de pailas, para que se encuentre entre $12-14 \mathrm{kgf} / \mathrm{cm}^{2}$ y se registre el diferencial de presión del tanque que se alinea a mercado.

Como resultado del estudio de R\&R se demostró que la carga por volumen a $90 \%$, por roto gauge, no mantiene los parámetros de repetibilidad por flujómetro, debido a que depende de la densidad, temperatura y el factor humano. Es conveniente realizar dicha carga por masa, ya que la medición es directa, prefijando el valor o la capacidad según las estadísticas del 2013 para cada carro.

\section{Etapa V: Controlar}

\section{Paso 16: Monitoreo del SISTEMA DE MEDICIÓN}

Para efectuar el monitoreo se utilizó nuevamente la carta EWMA para individuales, obteniendo la visualización del comportamiento de las mediciones realizadas durante el primer trimestre del año 2014 (figura 8).

Se observa que el instrumento continúa funcionando de manera estable y se encuentra bajo control estadístico.

\section{PAso 17: Cerrar y Difundir el PROYeCto de MejOra}

Se realizó la recopilación de los documentos empleados durante la investigación, redactando un documento final. Asimismo se plasmaron los principales logros después de poner en práctica las propuestas de mejora, entre las que sobresalen:

- Estudio de repetibilidad y estabilidad del flujómetro Coriolis en la entrega de GLP por camiones cisternas con la utilización del Máster Meter conectado en serie al instrumento durante el período en que se realiza el estudio, demostrándose que el mismo es estable en el tiempo y capaz de mantener sus características metrológicas.

- Estudio R\&R en la entrega de GLP regular por camiones rígidos, demostrando que la forma en que se realiza la entrega por volumen a $90 \%$ por roto gauge no permite garantizar condiciones de repetibilidad, pues está condicionado a la temperatura, densidad $\mathrm{y}$ al factor humano, donde es conveniente prefijar un valor promedio para cada carro, ya sea en volumen o en masa.

\section{Conclusiones}

Al término del trabajo se logra desarrollar y probar un procedimiento para la evaluación de calidad de las mediciones de flujo en procesos de transferencia fiscal en la entrega de GLP por cargadero de camiones en una empresa refinadora de petróleo, permitiendo ajustar la metodología seis sigma al estudio de las características metrológicas de un contador de flujo tipo Coriolis, fundamentalmente la estabilidad, repetibilidad y linealidad, demostrando que sus mediciones son confiables y que la causa principal de la variabilidad en las mediciones es el método de medición que no resultó ser el adecuado. Estos resultados permitieron proponer un conjunto de acciones para el tratamiento de las defi-

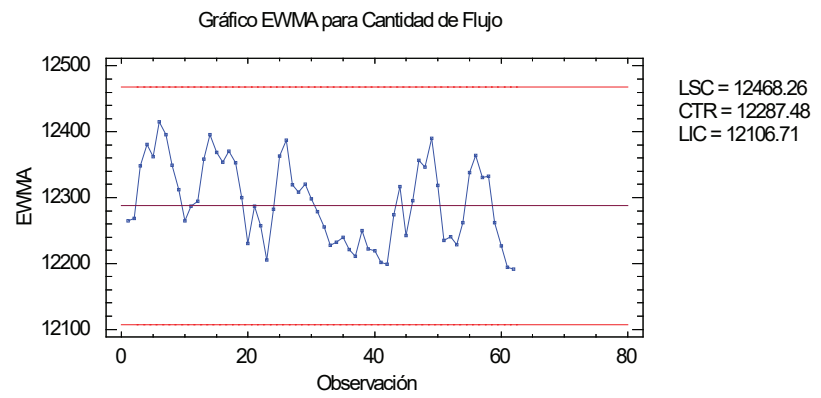

Figura 8. Carta EWMA para el volumen de GLP medido por el flujómetro Coriolis durante el primer trimestre del año 2014 
ciencias detectadas donde se implementaron y evaluaron parte de ellas, evidenciándose la necesidad de realizar la entrega por masa para disminuir la variabilidad otorgada por el factor humano, temperatura y densidad.

\section{RefERENCIAS}

Antony J. What does the future hold for quality professionals in organizations of the twenty-first century? The TQM Journal, volumen 25 (número 6), 2013: 677-685 [en línea] [fecha de consulta: 21 de enero de 2014]. Disponible en: http://www.emeraldinsight.com/journals.htm?articleid=17096596. ISSN 1754-2731.

Correa A. y Burgos C.M. Diseño e implementación de una metodología estadística para ensayos de producto terminado en Cementos El Cairo S.A. Revista Dyna, volumen 151, 2007: 195207 [en línea] [fecha de consulta: 21 de enero de 2014]. Disponible en: http://www.revistas.unal.edu.co/index.php/dyna/ article/viewFile/904/1315. ISSN 0012-7353.

Chang R. Estudio de mediciones en tanques de almacenamiento de hidrocarburos (tesis de maestría), Guatemala, Universidad de San Carlos de Guatemala. 2007.

European Association of National Metrology Institutes. Boletín de EURAMET (numero 8), 2013 [en línea] [fecha de consulta: 21 de enero de 2014]. Disponible en: www.euramet.org/.../ EURAMET_Newsletter_Issue_8_July_2013__Spanish _. pdf.

Franco J., Hugues-A., Estévez-A., Mendoza-R., Hernández-M., Sánchez-H. Proyecto de aseguramiento metrológico de las mediciones de productos claros en CUPET. Memorias del 9o Simposio Internacional Metrología, 2014.

Galvania L.R. y Carpinettib L.C. Análise comparativa da aplicação do programa Seis Sigma em processos de manufatura e serviços. Produção, volumen 23 (número 4), 2013: 695-704 [en línea] [fecha de consulta: 21 de enro de 2014]. Disponible en: http://www.scielo.br/pdf/prod/v23n4/aop_t6_0002_0489.pdf. ISSN 1300-3755.

Germanier M.L. y López-Carrizo M. Tecnologías para la medición en la transferencia de custodia, Memorias de la XVII Convención de Gas, 2006.

Gibbons P.M., Kennedy C., Burgess S., Godfrey P. The development of a value improvement model for repetitive processes (VIM). International Journal of Lean Six Sigma, volumen 3 (número 4), 2012: 315-338 [en línea] [fecha de consulta: 21 de enero de 2014]. Disponible en: http://www.emeraldinsight. $\mathrm{com} /$ journals.htm $?$ issn $=20404166 \&$ volume $=3 \&$ issue $=4 \&$ articl eid=17065659\&show=pdf . ISSN 2040-4166.

Guadalupe V.R. Diseño de una metodología a través de indicadores metrológicos que asegure los sistemas de medición en las industrias productoras de artículos plásticos, para mejorar la calidad de sus productos, (tesis de maestría), Escuela Superior Politécnica del Litoral, Guayaquil, Ecuador, 2008.
Gutiérrez H. y De la Vara R. Control estadístico de la calidad y Seis Sigma, México, Ed. McGraw-Hill, 2009, ISBN 970-10-4724-9.

Gutiérrez H. y De la Vara R. Análisis y diseño de experimentos, México, Ed. McGraw-Hill, 2008, ISBN 970-10-6526-3.

Hernández M. Aplicación de normas internacionales en la metrología legal para la medición de los combustibles en Cuba. Boletín Científico Técnico INIMET, 2012: 1-7.

Hernández M. Procedimiento para la mejora del sistema de gestión de las mediciones utilizando técnicas Seis Sigma en el proceso de tratamiento del turbo combustible Jet A1 en la Unidad de Negocio Refinería (tesis de maestría), Cienfuegos, Cuba, Universidad de Cienfuegos, 2012.

ISO 13053: 2011. Quantitative methods in process improvement, Six Sigma.

Machado F. Mejora de la gestión de las mediciones en el proceso de tratamiento y almacenamiento del turbo combustible Jet A1 en la Unidad de Negocio Refinería de Cienfuegos (tesis de grado), Cienfuegos, Cuba, Universidad de Cienfuegos, 2013.

Martínez T. Mejora del sistema de gestión de las mediciones en el proceso de entrega de gas licuado del petróleo regular en la Unidad de Negocio Refinería (tesis de grado), Cienfuegos, Cuba, Universidad de Cienfuegos, 2014

Montgomery D.C. Diseño y análisis de experimentos, México, Grupo Editorial Iberoamérica, 1991.

Myszewski J.M. On improvement story by 5 whys. The TQM Journal, volumen 25 (número 4), 2013: 371-383 [en línea] [fecha de consulta: 21 de enero de 2014]. Disponible en: http://www. emeraldinsight.com/journals.htm?issn=1754-2731\&volume=2 $5 \&$ issue $=4 \&$ articleid $=17090181 \&$ show $=$ abstract $\&$ PHPSESSID =ooabkr16arh0qpia25i8q47c60. ISSN 1754-2731.

NC-ISO 10012: 2007. Sistemas de gestión de las mediciones. Requisitos para los procesos de medición y los equipos de medición.

NC-ISO 9001.2015. Sistemas de gestión de la calidad, Requisitos.

NC 918: 2012. Metros Contadores para líquidos diferentes del agua. Métodos y equipos para la verificación.

Wang T. y Baker R. Coriolis flowmeters: a review of developments over the past 20 years, and an assessment of the state of the art and likely future directions. Flow Measurement and Instrumentation, volumen 40, 2014: 99-123.

Xiaofen T. Investigation on quality management maturity of Shanghai enterprises. The TQM Journal, volumen 25 (número 4), 2013: 417-430 [en línea] [fecha de consulta: 21 de enero de 2014]. Disponible en: http://www.emeraldinsight.com/journals.htm $?$ issn $=1754-2731 \&$ volume $=25 \&$ issue $=4 \&$ articleid $=170$ 90184\&show $=$ abstract\&PHPSESSID $=$ ofstj3gehli9495b16kg50p 6u2. ISSN 1754-2731.

Yvonne S. Statistical thinking in the quality movement \pm 25 years. The TQM Journal, volumen 25 (número 6), 2013: 597-605 [en línea] [fecha de consulta: 21 de enero de 2014]. Disponible en: h t t p: / / w w w.emeraldinsight.com/journals. htm? articleid=17096591. ISSN 1754-2731. 
Este artículo se cita:

Citación estilo Chicago

Barrera-García, Aníbal, Midiala Hernández-Santana, Leandro Oscar Botana-Beltrán, Tanayi Martínez-Hernández, Oleiny Omar CarrascoPadrón. Verificación de las características metrológicas de un contador de flujo Coriolis utilizado en transferencia fiscal. Ingeniería Investigación y Tecnología, XVIII, 04 (2017): 433-444.

\section{Citación estilo ISO 690}

Barrera-García A., Hernández-Santana M., Botana-Beltrán L., Martínez-Hernández T., Carrasco-Padrón O. Verificación de las características metrológicas de un contador de flujo Coriolis utilizado en transferencia fiscal. Ingeniería Investigación y Tecnología, volumen XVIII (número 4), octubre-diciembre 2017: 433-444.

\section{Semblanzas de los autores}

Aníbal Barrera-García. Ingeniero industrial, máster en ciencias en matemática aplicada, máster en ciencias en ingeniería industrial (Mención calidad). Profesor auxiliar del Departamento de Ingeniería Industrial de la Universidad de Cienfuegos. Imparte las asignaturas de metrología, sistemas integrados de gestión, ergonomía y seguridad y salud en el trabajo. Posee 10 años de experiencia.

Midiala Hernández-Santana. Ingeniera industrial, máster en ciencias en ingeniería industrial (Mención calidad). Especialista en metrología en la empresa CUVENPETROL S.A / Unidad de Negocios Refinería Cienfuegos. Posee más de 30 años de experiencia. Ha participado en múltiples eventos tanto nacionales como internacionales relacionados con la metrología.

Leandro Oscar Botana-Beltrán. Licenciado en física, máster en ciencias en energía y medio ambiente. Profesor auxiliar en el Departamento de Física de la Universidad de Cienfuegos. Imparte las asignaturas de física I, física II y física III para carreras de ingeniería. Posee más de 35 años de experiencia.

Tanayi Martínez-Hernández. Ingeniera industrial. Profesora instructora del Departamento de Ingeniería Industrial de la Universidad de Cienfuegos. Ha realizado investigaciones relacionadas con la metrología y ha participado en eventos científicos tanto nacionales como internacionales.

Oleiny Omar Carrasco-Padrón. Ingeniero informático. Profesor asistente del Departamento de Ingeniería Industrial de la Universidad de Cienfuegos. Imparte las asignaturas de bases de datos y programación. 